\title{
Residual Lifetime Prediction with Multistage Stochastic Degradation for Equipment
}

\author{
Zhan Gao, Qi-guo Hu $(\mathbb{D}$, and Xiang-yang Xu \\ Department of Mechatronics and Vehicle Engineering, Chongqing Jiaotong University, Chongqing 400074, China \\ Correspondence should be addressed to Qi-guo Hu; swpihqg@cqjtu.edu.cn
}

Received 26 September 2020; Revised 28 October 2020; Accepted 31 October 2020; Published 17 November 2020

Academic Editor: Wei Wang

Copyright (@) 2020 Zhan Gao et al. This is an open access article distributed under the Creative Commons Attribution License, which permits unrestricted use, distribution, and reproduction in any medium, provided the original work is properly cited.

\begin{abstract}
Residual useful lifetime (RUL) prediction plays a key role of failure prediction and health management (PHM) in equipment. Aiming at the problems of residual life prediction without comprehensively considering multistage and individual differences in equipment performance degradation at present, we explore a prediction model that can fit the multistage random performance degradation. Degradation modeling is based on the random Wiener process. Moreover, according to the degradation monitoring data of the same batch of equipment, we apply the expectation maximization (EM) algorithm to estimate the prior distribution of the model. The real-time remaining life distribution of the equipment is acquired by merging prior information of real-time degradation data and historical degradation monitoring data. The accuracy of the proposed model is demonstrated by analyzing a practical case of metalized film capacitors, and the result shows that a better RUL estimation accuracy can be provided by our model compared with existing models.
\end{abstract}

\section{Introduction}

Prognostics and health management (PMH) has been a systematic method utilized to evaluate the reliability or residual life of actual life-cycle conditions in a system, predict failure degree, reduce the risk in operating, improve task completion rate, and make maintenance decisions. Prognostics is usually characterized by estimating the RUL of a system based on condition monitoring $(\mathrm{CM})$ data $[1,2]$. Once a prognosis is available, some health management tactics, such as maintenance, replacement, and logistic support, can be performed properly to obtain the operational objectives, which is required $[3,4]$.

In general, RUL prediction is an important step in PMH. Over the last decade, there are a great deal of research studies as to RUL prediction have been developed for a large wide range of industrial products, for example, bearings $[5,6]$, gearboxes [7], lithium batteries [8], organic light emitting diodes [9], and laser generators [10]. The main idea of RUL is to realize the life prediction by obtaining the distribution or expectation of RUL according to the effective information such as equipment failure mechanism, CM data, and failure time data $[11,12]$. For fields with high safety and reliability requirements, for instance, aerospace and electronic equipment, whose performance gradually decreases as service time increases, their final failure is manifested as degraded failure. At the same time, due to measurement errors, environmental interference, and other factors, the degradation process has a certain degree of randomness. At present, life prediction based on statistical data is a central issue in current research $[13,14]$. Based on the probability density function (PDF) of the RUL with the randomness of the degradation process, the PDF of the RUL density can be obtained.

In life prediction based on statistical data, stochastic processes are often used to fit the degradation process, such as Gamma process $[15,16]$, Wiener process $[17,18]$, and Inverse Gaussian process $[19,20]$. The Wiener process is convenient to describe the degradation system that has a random nonmonotonic independent incremental process, which are influenced by external environment, internal state changes, and load conditions [21]. Li et al. in [22] proposed a novel RUL prediction models combined with degradation processes described by establishing the system age process 
and the system state process. They calculated the PDF of the RUL based on a closed-form solution by calculating the degradation trajectories values. Si et al. in [2] derived a random Wiener process model to predict the RUL of equipment by merging a recursive filtering algorithm and an EM algorithm for parameter estimation. With due consideration of the influence of measurement error, a nonlinear random Wiener model is proposed to predict remaining useful life [23].

The above research has enriched the application of stochastic processes in life prediction; however, a large number of the current degradation models made assumptions that the degradation process is subject to a single degradation law. Due to the staged changes in internal failure mechanism and dynamic changes in external environmental condition, many degradation process of equipment is characterized by multistage degradation [24]. However, for the current research in the Wiener process of the performance degradation modeling, there are few of them who target on multistage performance degradation models. Therefore, Wang et al. in [25] further proposed a two-stage degradation model of both Gamma process and Wiener process. In reference to the attenuation process of the brightness of light emitting diodes, Wang et al. in [9] assumed that individual differences exist in the change points and utilized a two-stage Wiener process model and Bayesian estimation method to analyze the degradation data under logarithmic transformation. In addition, how to integrate the degradation data of similar equipment to estimate the hyperparameters of the degradation model is the key to the RUL prediction. A two-stage maximum likelihood estimation (TSMLE) method was utilized to calculate the hyperparameters of the degradation model to achieve life prediction in some literatures [26]. Zhang et al. in [27] proposed a real-time residual life prediction algorithm including the Cumulative Sum (CUSUM) method and the Brownian motion with drift. However, the CUSUM method is only suitable for change-point degradation analysis and lacks the use of prior knowledge; when degradation data is small, the estimation accuracy of the two-step method is not high. Additionally, the change points in the multistage stochastic degradation process are sometimes scattered, so the RUL prediction based on the random process of the change point analysis is often not accurate enough.

Aiming at the above problems, in this paper, a multistage random degradation model based on the Wiener process is proposed to estimate the RUL of equipment aiming at above issues. Assuming that the parameters of the degradation model follow, respectively, certain random distributions to describe the differences between single equipment, an EM algorithm is used for iteratively estimating the prior distribution of the degradation model parameters based on the historical degradation data and historical failure time data. Finally, we apply the Bayesian algorithm to update the posterior distribution parameters of our model at each stage. By analyzing the degradation process of metallized film capacitors, the remaining life expectancy of individual capacitors can be predicted.

\section{Residual Life Prediction Model of Equipment}

2.1. Model Analysis and Assumptions. Generally, a degradation model of the Wiener process can be described as

$$
X(t)=u t+\sigma B(t),
$$

where $u$ is the drift coefficient, $\sigma$ is the diffusion coefficient, and $B(t)$ is the standard Brownian motion.

When we predict the remaining life of the equipment, the following assumptions should be made:

(1) If the performance degradation of the equipment exceeds the failure threshold, degradation failure will occur.

(2) The performance degradation of equipment has the characteristics of multiple stages, each performance degradation stage obeys the Wiener process, and it is divided by a certain fixed degradation amount.

(3) The drift coefficient $\mu$ and diffusion coefficient $\sigma$ of the Wiener process are random variables. We assume that $\omega=1 / \sigma^{2}$ and $f=(u, \omega)$ as joint prior distribution of $u$ and $\omega$, which obeys the GaussianGamma distribution. $f(u, \omega)$ can be expressed as

$$
\begin{aligned}
f(\mu, \omega)= & \frac{\omega^{1 / 2}}{\sqrt{2 \pi c}} \exp \left(\frac{-\omega(\mu-c)^{2}}{2 d}\right) \\
& \cdot \frac{b^{a}}{\Gamma(a)} \omega^{a-1} \exp (-b \omega),
\end{aligned}
$$

where $a, b, c$, and $d$ represent the hyperparameters in prior distribution of $u$ and $\omega$.

2.2. Performance Degradation Modeling. In fact, the degradation process of the equipment is characterized by multistage and randomness. A multistage degradation model of equipment can be expressed as

$$
\begin{aligned}
X(t)= & {\left[X(0)+X_{1}(t)\right] I_{\left(0, t_{1}\right)}(t)+\left[X\left(t_{1}\right)+X_{2}\left(t-t_{1}\right)\right] } \\
& \cdot I_{\left(t_{1}, t_{2}\right)}(t)+\cdots+\left[X\left(t_{n}\right)+X_{n}\left(t-t_{n}\right)\right] I_{\left(t_{n}, \infty\right)}(t),
\end{aligned}
$$

where $I(t)$ is the indicative function, $X(0)$ is the initial value of the Wiener degradation model, and $t_{i}$ represents the time that the performance degradation of each stage reaching its boundary value. $X_{k}(k=1,2, \ldots, n)$ is the performance degradation process of each stage.

The degradation process of the equipment in each stage can be described by using the Wiener process without loss of generality, so the performance degradation amount $X(t)$ of the equipment can be expressed as

$$
\begin{aligned}
X(t)= & {\left[X(0)+\mu_{1} t+\sigma_{1} B(t)\right] I_{\left(0, t_{1}\right)}(t)+\left[D_{1}+\mu_{2}\left(t-t_{1}\right)\right.} \\
& \left.+\sigma_{2} B\left(t-t_{1}\right)\right] I_{\left(t_{1}, t_{2}\right)}(t)+\cdots+\left[D_{n}+\mu_{n}\left(t-t_{n}\right)\right. \\
& \left.+\sigma_{n} B\left(t-t_{n}\right)\right] I_{\left(t_{n}, \infty\right)}(t),
\end{aligned}
$$


where $D_{k}$ is the boundary value of $k$ th performance degradation stage, $D_{n}$ represents the degradation failure threshold, and $\mu_{k}$ and $\omega_{k}$ are the drift coefficient and diffusion coefficient of the $k$ th stage of Wiener degradation, respectively.

The remaining life $L_{\tau}$ of the equipment represents the time from the performance degradation value $X(\tau)$ at current time $\tau$ to the failure threshold $D_{n}$ According to the definition of performance failure, the remaining life is expressed as

$$
L_{\tau}=\inf \left\{t: X(t+\tau) \geq D_{n}, t>0\right\} .
$$

Denote $T$ as the life of the equipment, according to the relationship between the life and the remaining life, and it can be expressed as

$$
T=\inf \left\{t: X(t) \geq D_{n}, t>0\right\}=L_{\tau}+\tau .
$$

Denote $\xi_{k}$ as the time when the device reaches the threshold value $D_{k}$ of the $k$ th stage of performance degradation from the initial moment, and $\xi_{k}$ can be expressed as

$$
\begin{aligned}
\xi_{k} & =\inf \left\{t: X(t) \geq D_{k}, t>0\right\}, \\
& =\xi_{k-1}+\inf \left\{t: X^{\prime}(t) \geq D_{k}-D_{k-1}, t>0\right\},
\end{aligned}
$$

where $X_{\prime}^{\prime}(t)=\mu_{k} t+\sigma_{k} B(t)$. Because the failure time of the Wiener process obeys the Inverse Gaussian distribution, $\xi_{k}$ obeys the Inverse Gaussian distribution $f_{\Delta \xi}\left(\Delta \xi \mid \mu_{k}, \omega_{k}\right)$, and it is formulated as

$$
f_{\Delta \xi}\left(\Delta \xi \mid \mu_{k}, \omega_{k}\right)=\frac{\omega_{k}^{1 / 2} \Delta D_{k}}{\sqrt{2 \pi\left(\Delta \xi_{k}\right)^{3}}} \exp \left(\frac{\omega_{k}\left(\Delta D_{k}-\mu_{k} \Delta \xi_{k}\right)^{2}}{2 \Delta \xi_{k}}\right) .
$$

Therefore, the probability density distribution function in the $k$ th stage degradation process of the equipment can be formulated as

$$
f\left(\Delta \xi_{k} \mid \mu_{k}, \omega_{k}\right)=\int_{0}^{\infty}\left(f_{\Delta \xi} \xi_{k} \mid \xi_{k-1}\right) \mathrm{d} \xi_{k}=\int_{0}^{\infty}\left(f_{\Delta \xi}\left(\xi_{k}-\xi_{k-1}\right)\right) \mathrm{d} \xi_{k} .
$$

When the performance degradation value $X(\tau)$ at $\tau$ of the equipment meets the condition $X\left(\xi_{k-1}\right) \leq X(\tau) \leq X\left(\xi_{k}\right)$, the remaining life of the degradation stage of equipment can be expressed as

$$
L_{\tau}=\left(\xi_{k}-\tau\right)+\Delta \xi_{k+1}+\Delta \xi_{k+2}+\cdots+\Delta \xi_{n} .
$$

The probability density distribution function of the remaining life $L_{\tau}$ of equipment degradation is expressed as

$$
\begin{aligned}
f_{L_{\tau}}\left(L_{\tau} \mid \mu_{k}, \omega_{k}, \mu_{k+1}, \omega_{k+1}, \ldots, \mu_{n}, \omega_{n}\right)= & \int_{\tau}^{\infty} \int_{\tau}^{\infty} \ldots \int_{\tau}^{\infty} f_{\Delta \xi}\left(l_{\tau}+\tau\right. \\
& \left.-\xi_{n-1}\right) f_{\Delta \xi}\left(\xi_{n-1}-\xi_{n-2}\right), \\
& \ldots, f_{\Delta \xi}\left(\xi_{k}-\tau\right) \mathrm{d} \xi_{k} \mathrm{~d} \xi_{k+1}, \\
& \ldots, \mathrm{d} \xi_{n-1} .
\end{aligned}
$$

Because of $E\left(\Delta \xi_{k}\right)=\Delta D_{k} / \mu_{k}$, the expectation of remaining life $L_{\tau}$ can be expressed as

$$
E\left(L_{\tau}\right)=\frac{W_{k}-X(\tau)}{\mu_{k}}+\frac{\Delta D_{k+1}}{\mu_{k+1}}+\cdots \frac{\Delta D_{n}}{\mu_{n}} .
$$

\section{Bayesian Deduction of Hyperparameters}

When the boundary value $D_{k}$ of each performance degradation stage is determined, $\mu$ and $\sigma$ of the Wiener process jointly determining the probability density distribution of the remaining life, the parameters $\mu$ and $\omega$ need to be solved. Because the degradation process of individual equipment is different, in order to describe it conveniently, we assume that $\mu$ and $\omega$ obey the following conjugate prior distribution:

$$
\begin{gathered}
\omega=\sigma^{-2} \sim \operatorname{Gamma}(a, b), \\
\mu \mid \omega \sim N\left(\frac{c, d}{\omega}\right) .
\end{gathered}
$$

When the real-time degradation data $X=\left(X_{1}, X_{2}, \ldots X_{n}\right)$ is obtained, we can utilize the Bayesian formula to derive the posterior distribution $\pi(\mu, \omega \mid X)$, which is formulated as

$$
\pi(\mu, \omega \mid X)=\frac{L(X \mid \mu t, n \omega) \cdot \pi(\mu, \omega)}{\int_{0}^{\infty} \int_{-\infty}^{\infty} L(X \mid \mu t, n \omega) \cdot \pi(\mu, \omega) \mathrm{d} \mu \mathrm{d} \omega},
$$

where $L(X \mid \mu t, n \omega)$ presents log-likelihood function for parameters $\mu$ and $\omega$, which is expressed as

$$
L(X \mid \mu t, n \omega)=\prod_{i=1}^{n} \frac{\omega^{1 / 2}}{\sqrt{2 \pi \Delta \Lambda\left(t_{i}\right)}} \exp \left(\frac{-\omega\left(\Delta X_{i}-\mu \Delta \Lambda\left(t_{i}\right)\right)^{2}}{2 \Delta \Lambda\left(t_{i}\right)}\right) .
$$

$\pi(\mu, \omega)$ is the joint prior density function of $\mu$ and $\omega$, which is formulated as

$$
\pi(\mu, \omega)=\frac{\omega^{1 / 2}}{\sqrt{2 \pi d}} \exp \left(\frac{-\omega(\mu-c)^{2}}{2 d}\right) \frac{b^{a} \omega^{a-1}}{\Gamma(a)} \exp (-b \omega) .
$$


In view of the derivation above, we combine equations (14)-(16) to obtain the following form:

$$
\begin{array}{r}
\pi(\mu, \omega \mid X) \propto L(X \mid \mu, \omega) \cdot \pi(\mu, \omega) \propto \omega^{(n+1) / 2+a-1} \cdot \exp \left\{-\frac{\omega}{2}\left(\left(\mu^{2} \Lambda\left(t_{n}\right)-2 \mu X_{n}+\sum_{i=1}^{n} \frac{\Delta X_{i}^{2}}{\Delta \Lambda\left(t_{i}\right)}\right)-\frac{\omega}{2}\left(\frac{(\mu-c)^{2}}{d}\right)-b \omega\right) \propto\right. \\
\omega^{(n+1) / 2+a-1} \exp \left\{-\omega\left(b+\frac{c^{2}}{2 d}-\frac{\left(X_{n} d+c\right)^{2}}{2\left(\Lambda\left(t_{n}\right) d^{2}+d\right)}\right)+\sum_{i=1}^{n} \frac{\Delta X_{i}^{2}}{2 \Delta \Lambda\left(t_{i}\right)}\right\} \cdot \omega^{1 / 2} \exp \left\{\frac{\omega}{2}\left(\frac{\left(\mu-X_{n} d+c / \Lambda\left(t_{n}\right) d+1\right)^{2}}{d / \Lambda\left(t_{n}\right) d+1}\right)\right\} .
\end{array}
$$

Because the conjugate prior distribution of random parameters and its posterior distribution have the same distribution function, the difference is only the change of the hyperparameter value in different degradation stages. According to equation (17), we can obtain the posterior estimation values $a_{k}^{\prime}, b_{k}^{\prime}, c_{k}^{\prime}$, and $d_{k}^{\prime}$ of the hyperparameters in the $k$ th performance degradation stage deduced as

$$
\left\{\begin{array}{l}
a_{k}^{\prime}=\frac{n_{k}}{2}+a_{k}, \\
b_{k}^{\prime}=b_{k}+\frac{c_{k}^{2}}{2 d_{k}}-\frac{\left(X_{n, k} d_{k}+c_{k}\right)^{2}}{2\left(\Lambda\left(t_{n, k}\right) d_{k}^{2}+d_{k}\right)}+\sum_{i=1}^{n} \frac{\Delta X_{i, k}^{2}}{2 \Delta \Lambda\left(t_{i, k}\right)} \\
c_{k}^{\prime}=\frac{X_{n, k} d_{k}+c_{k}}{\Lambda\left(t_{n, k}\right) d_{k}+1} \\
d_{k}^{\prime}=\frac{d_{k}}{\Lambda\left(t_{n, k}\right) d_{k}+1} .
\end{array}\right.
$$

The posterior mean $\mu^{\prime}$ and $\omega^{\prime}$ of $\mu$ and $\omega$ can be obtained from $a^{\prime}, b^{\prime}, c^{\prime}$, and $d^{\prime}$, and from equation (17), the $k t h$ performance degradation stage is expressed as

$$
\begin{aligned}
\pi\left(\mu_{k}, \omega_{k} \mid t \Delta n X_{k}\right)= & \frac{b_{k}^{\prime} a_{k}^{\prime} \omega_{k}^{a_{k}^{\prime}-1}}{\Gamma\left(a_{k}^{\prime}\right)} \exp \left(-\omega_{k} b_{k}^{\prime}\right) \\
& \cdot \frac{\omega_{k}^{\prime}}{\sqrt{2 \pi d_{k}^{\prime}}} \exp \left(-\frac{\omega_{k}\left(\mu_{k}-c_{k}^{\prime}\right)^{2}}{\sqrt{2 \pi d_{k}^{\prime}}}\right) .
\end{aligned}
$$

After getting the result of $\pi\left(\mu_{k}, \omega_{k} \mid t \Delta n X_{k}\right)$, we can obtain the posterior mean values $\mu_{k}^{\prime}$ and $\omega_{k}^{\prime}$ in $k$ th stage to realize the prediction of the remaining life of the equipment.

\section{Parameter Estimation of Degradation Model}

4.1. Determination of the Complete Maximum Likelihood Function of the Hyperparameters. When the boundary value of each stage of performance degradation is determined, the probability density distribution of the remaining life $L_{\tau}$ can be determined by the Wiener process parameters $\mu$ and $\omega$ in each performance degradation stage. Therefore, parameters $\mu$ and $\omega$ is required to estimate. Because we assumed that the unknown parameters $\mu$ and $\omega$ are random variables, the unknown hyperparameters in the prior distribution of parameters can be estimated based on the historical performance degradation data and historical failure time data [28].

For the same kind of equipment, we can collect the degradation data of $N$ equipments as priori information in $\mathrm{CM}$ time. Assuming that the performance degradation data of the $i$ th $(i=1,2, \ldots, N)$ equipment at the $j$ th $(j=1,2, \ldots, n)$ measurement is denoted as $X_{i j}$, the corresponding measurement time is $t_{i j}$. The $k \Delta x_{k}$ and $\Delta t_{k}$ of the historical data sample of the $k$ th performance degradation stage are, respectively, expressed as

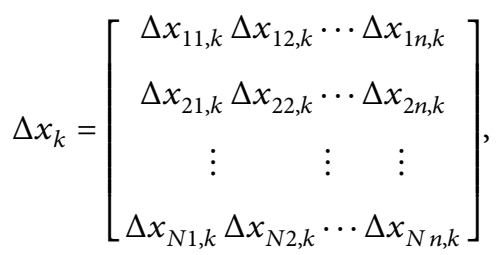

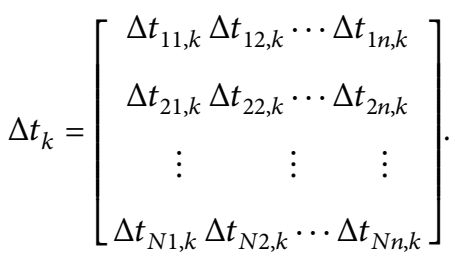

The probability density function of the degradation increment $\Delta X_{k}(t)$, in the $k$ th stage of degradation based on the characteristics of the Wiener process, can be expressed as

$$
f\left(\Delta X_{k}\right)=\frac{1}{\sqrt{2 \pi \Delta t_{k} \sigma_{k}^{2}}} \exp \left[-\frac{\left(\Delta X_{k}-\mu_{k} \Delta t_{k}\right)}{2 \sigma_{k}^{2} \Delta t_{k}}\right] .
$$

Therefore, the complete likelihood functions of hyperparameters $a_{k}, b_{k}, c_{k}$, and $d_{k}$ of the historical degradation data $t_{i j, k}, X_{i j, k}$ in the $k$ th stage are expressed as 


$$
\begin{aligned}
L\left(\Delta X_{k} \mid a_{k}, b_{k}, c_{k}, d_{k}\right)= & \prod_{i=1}^{N} \prod_{j=1}^{n} h\left(\Delta X_{i j, k}\right) \cdot \prod_{i=1}^{N} \pi\left(\mu_{i, k}, \omega_{i, k}\right) \\
= & \prod_{i=1}^{N} \prod_{j=1}^{n} \frac{\omega_{i, k}^{1 / 2}}{\sqrt{2 \pi \Delta t_{i j, k}}} \\
& \cdot \exp \left[-\frac{\omega_{i, k}\left(\Delta X_{i j, k}-\mu_{i, k} \Delta t_{i j, k}\right)^{2}}{2 \Delta t_{i j, k}}\right] \cdot \prod_{i=1}^{N} \\
& \cdot \frac{b_{k}^{a_{k}} \omega_{i, k}^{a_{k}-1}}{\Gamma\left(a_{k}\right)} \exp \left(-\omega_{i, k} b_{k}\right) \\
& \cdot \frac{\omega_{i, k}^{1 / 2}}{\sqrt{2 \pi d_{k}}} \exp \left[-\frac{\omega_{i, k}\left(\mu_{i, k}-c_{k}\right)^{2}}{2 d_{k}}\right] .
\end{aligned}
$$

In theory, we can obtain the priori estimates of hyperparameters for the $k$ th stage Wiener process $\widehat{a}_{k}, \widehat{b}_{k}, \widehat{c}_{k}$, and $\hat{d}_{k}$ through the traditional maximum likelihood estimation (MLE), and it can be expressed as

$$
\left\{\begin{array}{l}
\psi\left(\widehat{a}_{k}\right)-\ln \widehat{a}_{k}=\sum_{i=1}^{N} \ln \frac{\omega_{i, k}}{N}+\ln N-\ln \sum_{i=1}^{N} \omega_{i, k}, \\
\widehat{b}_{k}=N \cdot \frac{\widehat{a}_{k}}{\sum_{i=1}^{N} \omega_{i, k}}, \\
\widehat{c}_{k}=\sum_{i=1}^{N} \mu_{i, k} \omega_{i, k}, \\
\widehat{d}_{k}=\sum_{i=1}^{N} \frac{\left(\omega_{i, k} \mu_{i, k}^{2}-2 \widehat{c}_{k} \mu_{i, k} \omega_{i, k}+\widehat{c}_{k}^{2} \omega_{i, k}\right)}{N}
\end{array}\right.
$$

where $\psi(\cdot)$ is the digamma distribution function, $\mu_{i, k}$ and $\omega_{i, k}$ are implicit data, and the unknown items of the implicit data must be output first, otherwise, we cannot obtain the results of $\widehat{a}_{k}, \widehat{b}_{k}, \widehat{c}_{k}$, and $\widehat{d}_{k}$ directly.

4.2. Estimation of Hyperparameter. The EM algorithm is an effective method for maximum likelihood estimation [29]. It is mainly used for parameter estimation of missing data, for instance, the EM algorithm can be applied to estimate the parameters of likelihood function when observed data is incomplete or the expression of the likelihood function is so complicated that the traditional estimation method of the maximum likelihood function could be failure.

Each recursive iteration process of the EM algorithm contains $E$ steps and $M$ steps. The first step of the EM algorithm (E-step): given the observed data $\Delta X_{k}$ and current hyperparameter $a_{k}, b_{k}, c_{k}$, and $d_{k}$ estimated value, we can get the conditional expected value of unknown latent variable $Z=(\mu, \omega)$ in complete data log-likelihood function $L\left(\Delta X_{k} \mid a_{k}, b_{k}, c_{k}, d_{k}\right)$. The second step of the EM algorithm (M-step): solve the $a_{k}, b_{k}, c_{k}$, and $d_{k}$ and make them as parameter estimate values for the next iteration.

In $E$-step, if iteration process performs $r$ times in the $k$ th stage of the degradation process, $a_{l, k}, b_{l, k}, c_{l, k}$, and $d_{l, k}$ are the estimated value in $l$ th $(1 \leq l \leq r)$. The expectation that each unknown item contains implicit data $\mu_{i, k}, \omega_{i, k}$ in the $l+1$ step iteration process is

$$
\begin{aligned}
E\left(\omega_{i, k}\right) & =\frac{\widehat{a}_{l, k}+N_{i} / 2}{\widehat{b}_{l, k}+\widehat{c}_{l, k}^{2} / 2 \widehat{d}_{l, k}-\left(\widehat{d}_{l, k} X_{i N_{i}, k}+\widehat{c}_{l, k}\right)^{2} / 2 \widehat{d}_{l}\left(t_{i N_{i}, k} \widehat{d}_{l, k}+1\right)+\sum_{j=1}^{N_{i}} \Delta X_{i j, k}^{2} / 2 \Delta \Lambda\left(t_{i j, k}\right)}, \\
E\left(\ln \omega_{i, k}\right) & =\psi\left(\widehat{a}_{l, k}+N_{i} / 2\right)-\ln \left(\widehat{b}_{l, k}+\widehat{c}_{l, k}^{2} / 2 d_{l, k}-\left(d_{l, k} X_{i N_{i}, k}+\widehat{c}_{l, k}\right)^{2} / 2 d_{l, k}\left(d_{l, k} t_{i N_{i}, k}+1\right)+\sum_{j=1}^{N_{i}} \Delta X_{i j, k}^{2} / 2 t_{i j, k}\right), \\
E\left(\omega_{i, k}, \mu_{i, k}\right) & =E\left(\omega_{i, k}\right) \cdot \frac{\widehat{d}_{l, k} X_{i N_{i}, k}+\widehat{c}_{l, k}}{t_{i N_{i}, k} \widehat{d}_{l, k}+1}, \\
E\left(\omega_{i, k}, \mu_{i, k}^{2}\right) & =E\left(\omega_{i, k}\right) \cdot\left(\frac{\widehat{d}_{l, k} X_{i N_{i}, k}+\widehat{c}_{l, k}}{t_{i N_{i}, k} \widehat{d}_{l, k}+1}\right)^{2}+\frac{\widehat{d}_{l, k}}{t_{i N_{i}, k} \widehat{d}_{l, k}+1} .
\end{aligned}
$$


In $M$-step, we substitute the expectations of $\mu_{i, k}$ and $\omega_{i, k}$ that are obtained in $E$-step into equation (23), and the estimated values of hyperparameters $\widehat{a}_{l+1}, \widehat{b}_{l+1}, \widehat{c}_{l+1}$, and $\widehat{d}_{l+1}$ after $l+1$ iterations under the $k$ th stage degradation can be obtained as

$$
\left\{\begin{array}{l}
\psi\left(\widehat{a}_{l+1, k}\right)-\ln \widehat{a}_{l+1, k}=\sum_{i=1}^{N} E \frac{\left(\ln \omega_{i, k}\right)}{N}+\ln N-\ln \sum_{i=1}^{N} E\left(\omega_{i, k}\right), \\
\widehat{b}_{l+1, k}=N \cdot \frac{\widehat{a}_{l+1, k}}{\sum_{i=1}^{N} E\left(\omega_{i, k}\right)}, \\
\widehat{c}_{l+1, k}=\frac{\sum_{i=1}^{N} E\left(\omega_{i, k} \mu_{i, k}\right)}{\sum_{i=1}^{N} E\left(\omega_{i, k}\right)}, \\
\widehat{d}_{l+1, k}=\frac{\sum_{i=1}^{N}\left(E\left(\omega_{i, k} \mu_{i, k}^{2}\right)-2 \widehat{c}_{l+1, k} E\left(\omega_{i, k} \mu_{i, k}\right)+\widehat{c}_{l+1, k}^{2} E\left(\omega_{i, k}\right)\right)}{N} .
\end{array}\right.
$$

If the error between $\widehat{a}_{r-1}, \widehat{b}_{r-1}, \widehat{c}_{r-1}$, and $\widehat{d}_{r-1}$ and $\widehat{a}_{r}, \widehat{b}_{r}$, $\widehat{c}_{r}$, and $\widehat{d}_{r}$ reaches the set condition after $r$ iterations, then the a priori estimate of the hyperparameter is obtained.

\subsection{Real-Time Remaining Life Distribution of Equipment.} If joint posterior distribution of the parameters $\mu$ and $\omega$ are obtained, the marginal probability distributions can be obtained, respectively. Because the joint posterior distribution of $\mu$ and $\omega$ obeys Gaussian-gamma distribution, the posterior distribution of $\mu$ obeys $\mu \sim t\left(2 a^{\prime} d^{\prime}\right)$ and the posterior distribution of $\omega$ obeys $1 / \omega^{\prime} \sim \operatorname{IG}\left(a^{\prime}, 1 / b^{\prime}\right)$. IG $(\cdot)$ is the Inverse Gamma distribution, so the posterior distribution of $\mu$ and $\omega$ can be expressed as

$$
\left\{\begin{array}{l}
\mu_{k}=E\left(\mu_{k} \mid t \Delta n X_{k}\right)=d_{k}^{\prime}, \\
\omega_{k}=E\left(\omega_{k} \mid t \Delta n X_{k}\right)=\frac{a_{k}^{\prime}}{b_{k}^{\prime}} .
\end{array}\right.
$$

Moreover, the real-time remaining life density distribution of the equipment can be further expressed as

$$
f_{L_{\tau}}\left(L_{\tau}\right)=f_{L_{\tau}}\left(L_{\tau} \mid t \widehat{\mu}_{1} n, q \widehat{\omega}_{1} h, \widehat{\mu}_{2} x, 7 \widehat{\omega}_{2} C, ; \ldots, \widehat{\mu}_{k}, \widehat{\omega}_{k}\right) .
$$

4.4. Prediction Steps for Remaining Life of Single Equipment. When the real-time performance degradation data of a single equipment is obtained, the remaining life of a single aero engine can be predicted in real time, and the specific steps are as follows:

Step 1 : we estimate the unknown hyperparameters in the prior distribution of the parameters $\mu$ and $\omega$ of the degradation model at each stage, getting estimated values $\widehat{a}, \widehat{b}, \widehat{c}$, and $\widehat{d}$ by the EM algorithm by historical degradation data $X$ and failure time data $\xi$.

Step 2 : when the real-time performance degradation data $\Delta X$ of the equipment is obtained, the model parameters in each performance degradation stage are updated in real time using equation (18) according to the Bayesian method. Moreover, we get the estimated hyperparameter values $\hat{a}^{\prime}, \widehat{b}^{\prime}$, $\widehat{c}^{\prime}$, and $\widehat{d}^{\prime}$ in posterior distribution in our model.

Step 3 : according to equation (26) the posterior estimated values $\widehat{\mu}$ and $\widehat{\omega}$ of our model in each performance degradation stage are calculated, respectively, and substituting them into equation (27), the remaining life density distribution of equipment after Bayesian updating can be obtained. Finally, we can estimate the expectancy value of equipment remaining life though calculating equation (12).

\section{A Practical Case Study}

Metalized film capacitor, as one of an important component in Inertial Confinement Fusion (ICF) laser equipment, is the key of life prediction to maintain the laser equipment and to develop a spare parts strategy [30]. However, some problems are unavoidable such as impurity or defect areas in organic film of a metallized film capacitor when it is in the production process. It is prone to form a discharge channel broken down by the external voltage during multiple charge and discharge processes, and because of weak electrical resistance in areas, capacitor result in "self-healing" phenomenon. A single self-healing causes a very small loss of capacitance; however, as the cycle of charge and discharge process increases, the capacitance value of the capacitor decreases continuously. When the loss of capacitance exceeds a certain threshold, the degradation rate of capacitance increases. Usually, we define $5 \%$ of the capacity loss as the end-of-life index [31]. The variation of the capacitance degradation under normal working conditions of the eight capacitors is shown in Figure 1, and $t$ is the number of charge and discharge.

It can be observed from Figure 1 that the capacitance of most capacitors shows a decreasing trend at the increase of the number of charge and discharge. The degradation rate of metallized film capacitors is relatively slow in the initial stage. When the capacitance loss reaches about $0.23 \mu \mathrm{F}$, the capacitance degradation speed accelerates. We assume that the performance degradation threshold $D_{1}=0.23$. Degradation of another capacitor during working of charge and discharge is shown in Table 1 , and we record this capacitor as No. 9 capacitor. Degradation failure occurs when the capacitance change $\Delta C$ of the capacitor exceeds $5 \%$ of the initial capacitance value $C_{0}$. We record the failure threshold $D_{n}=5 \% C_{0}$.

Take degradation data in first five of the 8 capacitors as historical monitoring data for priori parameter estimation, 


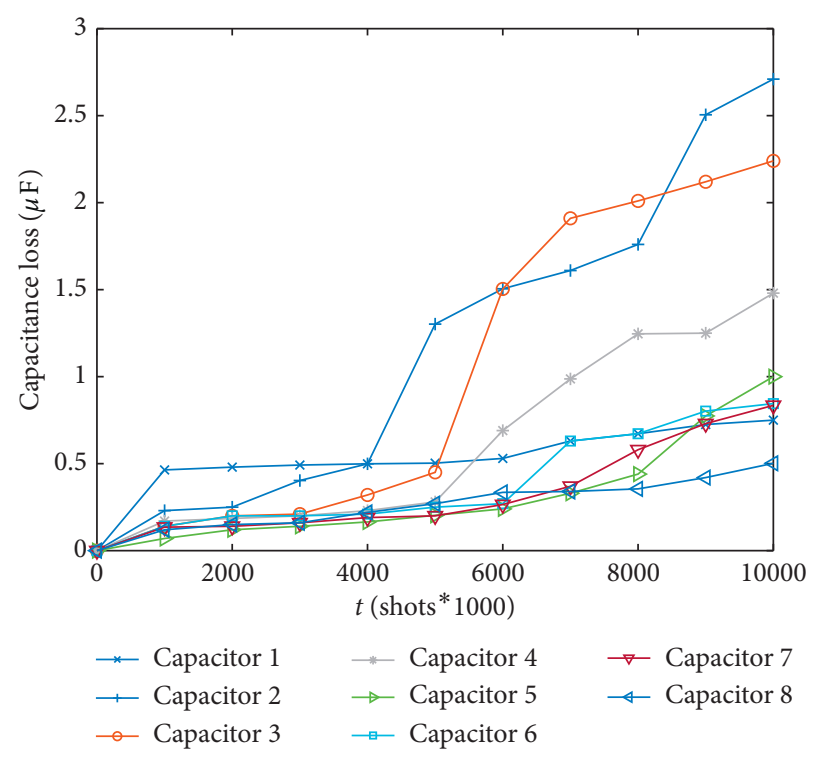

FIgUre 1: Capacitance degradation curves.

TABle 1: Capacitance of No. 9 capacitor.

\begin{tabular}{lcccc}
\hline$t /$ shots & 0 & 1000 & 2000 & 3000 \\
\hline$C(t) / \mu \mathrm{F}$ & 56.71 & 56.68 & 56.64 & 56.56 \\
$t /$ shots & 4000 & 5000 & 6000 & 7000 \\
$C(t) / \mu \mathrm{F}$ & 56.38 & 56.10 & 56.06 & 55.82 \\
\hline
\end{tabular}

and take the degradation data in last three of them as realtime data for posterior parameter updating accordingly. We assume that the initial values of the hyperparameter is $(1,1$, $1,1)$, and when the error between the adjacent estimated values after iteration is not greater than $10^{-6}$ to end EM iteration process, the priori estimated hyperparameter values $\widehat{a}, \widehat{b}, \widehat{c}$, and $\widehat{d}$ obtained. Moreover, the iterative convergence process of hyperparameter estimation is shown in Figure 2, and the a priori estimated values of hyperparameters at each stage are shown in Table 2.

After determining the prior distribution of the model parameters in each performance degradation stage, the realtime performance degradation data of a single capacitor is obtained. Moreover, according to the Bayesian method, the model parameters in each performance degradation stage can be updated in real time. Take No. 9 capacitor as an example for life prediction, and we rely on real-time performance degradation data of last three of capacitors to estimate the hyperparameters of the posterior distribution in the first stage and second stage. Tables 3 and 4 show the estimated values after Bayesian updating in different stages. Finally, we substitute the estimated values of Bayesian updating in the first stage and second stage into equation (27), and the probability density distribution of the remaining life can be obtained. Figures 3 and 4 show the real-time remaining life probability density distribution diagrams of No. 9 capacitor in the first and second stages, respectively.
The EM algorithm has certain advantage in estimation of hyperparameter. Comparing the EM algorithm with the Two-stage Maximum Likelihood Estimation (TSMLE) [26] for the error of the hyperparameter estimation results, we utilize the sum of relative errors Err of 4 hyperparameter estimations to show the precision of the estimation results, and Err is expressed as

$$
\operatorname{Err}=\frac{|\widehat{a}-t a|}{a}+\frac{|\widehat{b}-t b|}{b}+\frac{|\widehat{c}-t c|}{c}+\frac{|\widehat{d}-t d|}{d} .
$$

The estimation error between the two methods is shown in Table 5. The result of Err in the EM algorithm and the TSMLE method can be seen. Obviously, the EM algorithm has certain accuracy advantage in the estimation of hyperparameters.

Table 6 shows the Relative Error (RE) of RUL prediction for No.9 Capacitor in our model, model of single-stage degradation [31] and the model of Brownian motion with drift [32]. It can be seen that the relative error of considering the multistage degradation process is smaller than the other models, which proves that the proposed model can provide better accuracy for predicting the remaining life of metallized film capacitors. In addition, due to environmental influence, defects, or impurities of organic film in the same type capacitors, individual capacitor has different degradation. Therefore, the Wiener process distribution parameter estimation based on Bayesian estimation of prior 

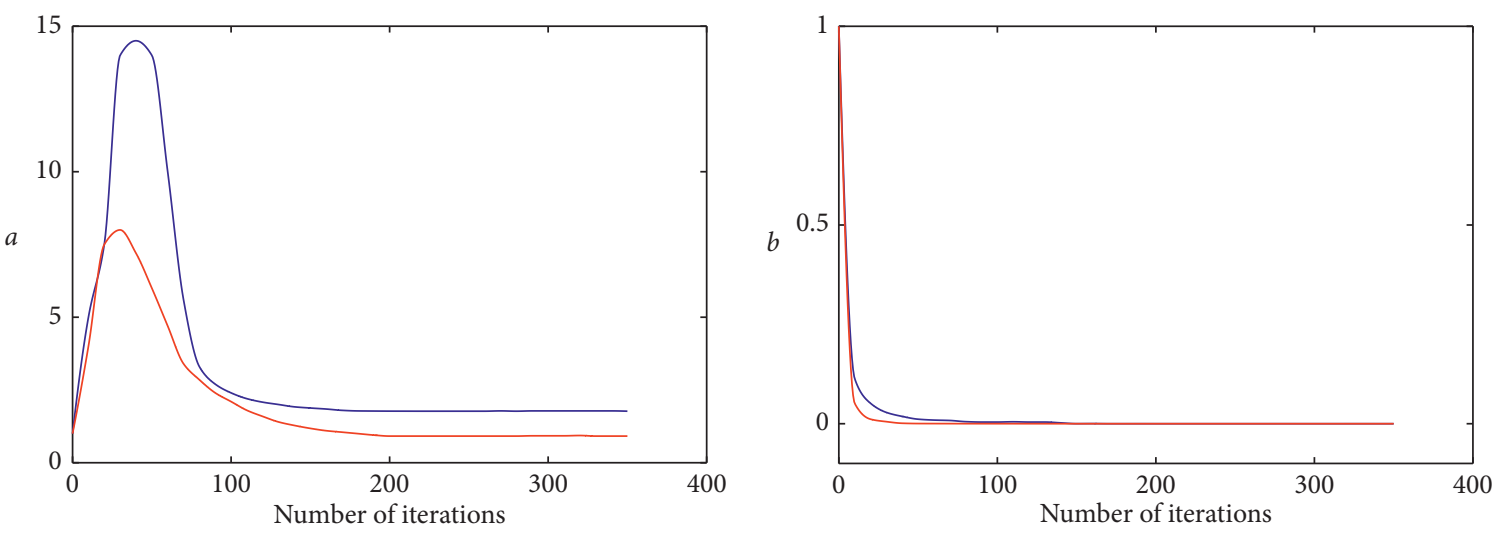

$$
-a_{1}
$$

(a)

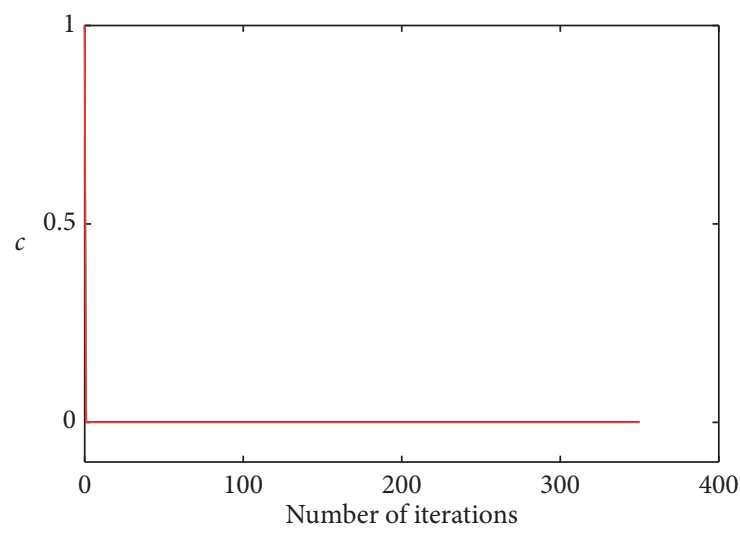

$c_{1}$
$c_{2}$ $b_{1}$

$-b_{2}$

(b)

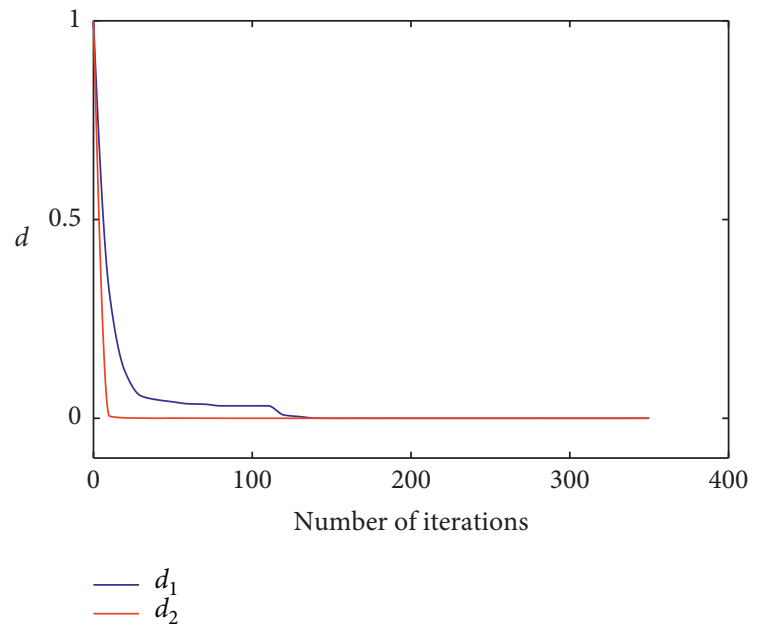

(d)

FIGURE 2: Iterative convergence process of hyperparameter estimation.

TABLE 2: Estimation of hyperparameter in prior distribution.

\begin{tabular}{lcccc}
\hline Stage & \multicolumn{3}{c}{ Estimation value of posterior } \\
& $a$ & $b\left(10^{-5}\right)$ & $c\left(10^{-5}\right)$ & $d\left(10^{-4}\right)$ \\
\hline 1 & 1.7710 & 1.1365 & 3.1618 & 0.7612 \\
2 & 0.9153 & 2.0641 & 5.7205 & 1.6162 \\
\hline
\end{tabular}

TABLE 3: Updated hyperparameters based on the Bayesian method (1st stage).

\begin{tabular}{|c|c|c|c|c|}
\hline \multirow{2}{*}{ Estimation value of posterior } & \multicolumn{4}{|c|}{$t /$ shots } \\
\hline & 1000 & 2000 & 3000 & 4000 \\
\hline$a^{\prime} 1\left(10^{-5}\right)$ & 1.7710 & 2.2710 & 2.7710 & 0.9071 \\
\hline$b^{\prime} 1\left(10^{-5}\right)$ & 1.3020 & 1.0007 & 0.9785 & 2.0572 \\
\hline$c^{\prime} 1\left(10^{-5}\right)$ & 3.1618 & 3.1128 & 3.0862 & 5.6825 \\
\hline$d^{\prime} 1\left(10^{-4}\right)$ & 0.7612 & 0.7274 & 0.6606 & 1.6197 \\
\hline $\mathrm{RL}\left(10^{4} /\right.$ shot $)$ & 2.832 & 2.724 & 2.603 & 2.470 \\
\hline
\end{tabular}


TABLE 4: Updated hyperparameters based on the Bayesian method (2nd stage).

\begin{tabular}{lccc}
\hline Estimation value of posterior & \multicolumn{3}{c}{ t/shots } \\
& 5000 & 6000 & 7000 \\
\hline$a^{\prime} 2$ & 0.9353 & 1.4153 & 1.9153 \\
$b^{\prime} 2$ & 2.0641 & 2.0587 & 2.0529 \\
$c^{\prime} 2$ & 5.7178 & 5.6421 & 5.5129 \\
$d^{\prime} 2$ & 1.6162 & 1.6098 & 1.6103 \\
$\mathrm{RL}\left(10^{4} /\right.$ shot $)$ & 2.402 & 2.224 & 2.178 \\
\hline
\end{tabular}

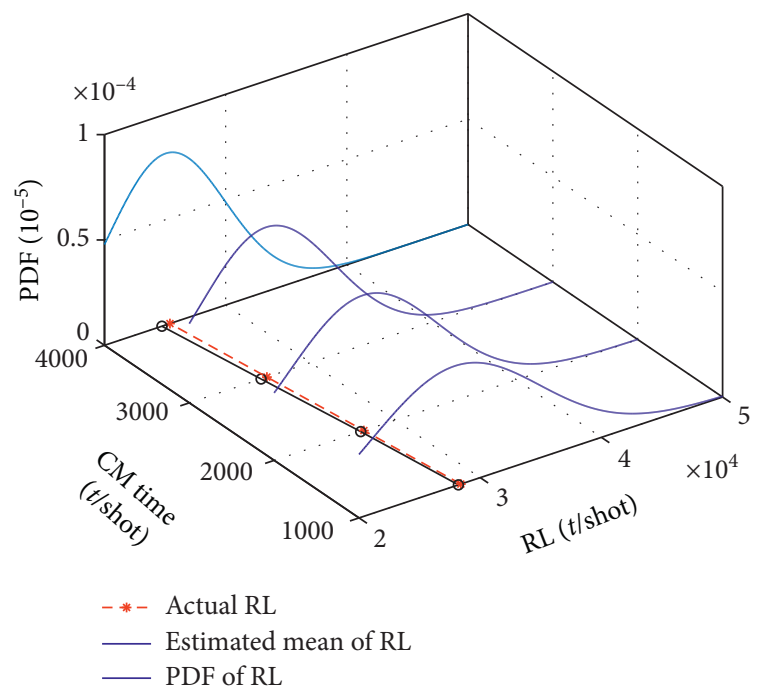

FIGURE 3: Probability density distribution of residual life (first stage).

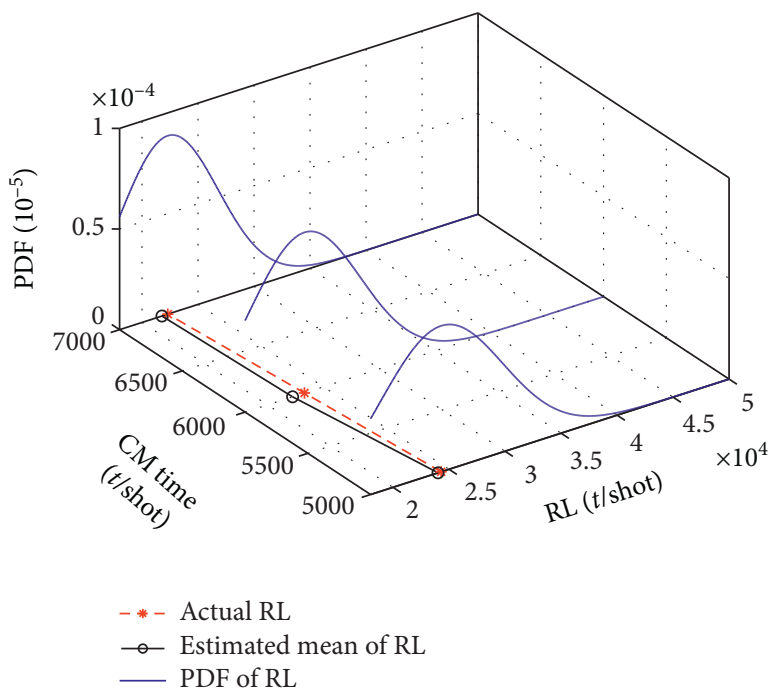

Figure 4: Probability density distribution of residual life (second stage).

TABle 5: Comparison of error for the estimation value of hyperparameters.

\begin{tabular}{lccc}
\hline Stage & \multicolumn{2}{c}{ Err } \\
& EM & & TSMLE \\
\hline 1 & 0.0891 & 0.2005 \\
2 & 0.1278 & 0.4503 \\
\hline
\end{tabular}

TABle 6: RE of RUL prediction for No. 9 Capacitor.

\begin{tabular}{lccc}
\hline $\begin{array}{l}\text { CM time } \\
(t / \text { shot })\end{array}$ & $\begin{array}{c}\text { Model of } \\
\text { single-stage } \\
\text { degradation }\end{array}$ & $\begin{array}{c}\text { Our } \\
\text { model }\end{array}$ & $\begin{array}{c}\text { Model of } \\
\text { Brownian } \\
\text { motion with drift }\end{array}$ \\
\hline 1000 & 0.0120 & 0.0097 & 0.0086 \\
2000 & 0.0195 & 0.0135 & 0.0142 \\
3000 & 0.0219 & 0.0203 & 0.0224 \\
4000 & 0.0454 & 0.0312 & 0.0497 \\
5000 & 0.0268 & 0.0127 & 0.0264 \\
6000 & 0.0405 & 0.0340 & 0.0435 \\
7000 & 0.0304 & 0.0264 & 0.0312 \\
\hline
\end{tabular}

degradation information is more in line with engineering reality.

\section{Conclusion Remarks}

In this paper, we propose a degradation model based on the Wiener process combined with the EM algorithm and Bayesian method to estimate the RUL of equipment. A RUL estimation method is constructed with due considering the multistage degradation and individual differences in equipment. By assuming that parameters of the Wiener process are random variables which obey a certain distribution, we utilize EM algorithm to estimate prior parameter of the model based on historical degradation monitoring data and updating posterior parameters based on CM data by Bayesian. Finally, we can obtain different CDF of RUL and expectation value of RUL.

By comparing the proposed model with existing models, we utilize RE to measure prediction accuracy, and the result shows that the accuracy of proposed model is better than existing models. The Wiener process that considers individual differences can better describe the degradation process of the equipment, and multistage degradation is more suitable in engineering practical. In addition, we can see that the estimation accuracy of EM is better than the TSMLE method in hyperparameter estimation.

\section{Data Availability}

The data used to support the findings of this study are available from the corresponding author upon request.

\section{Conflicts of Interest}

The authors declare that they have no conflicts of interest.

\section{References}

[1] Y. Peng and M. Dong, "A prognosis method using age-dependent hidden semi-markov model for equipment health prediction," Mechanical Systems and Signal Processing, vol. 25, no. 1, pp. 237-252, 2011.

[2] X. S Si, W. Wang, C. Hu, and D.-H. Zhou, "A Wiener-processbased degradation model with a recursive filter algorithm for remaining useful life estimation," Mechanical Systems and Signal Processing, vol. 35, no. 1-2, pp. 219-237, 2013. 
[3] X.-S. Si, W. Wang, C.-H. Hu, and D.-H. Zhou, "Remaining useful life estimation - a review on the statistical data driven approaches," European Journal of Operational Research, vol. 213, no. 1, pp. 1-14, 2011.

[4] M.-Y. You, F. Liu, W. Wang, and G. Meng, "Statistically planned and individually improved predictive maintenance management for continuously monitored degrading systems," IEEE Transactions on Reliability, vol. 59, no. 4, pp. 744-753, 2010.

[5] Y. Hu, P. H. Li, K. Z. WangChai, and Z. Chen, "A prediction method for the real-time remaining useful life of wind turbine bearings based on the wiener process," Renewable Energy, vol. 127, pp. 452-460, 2018.

[6] L. Xie, "A recurrent neural network based health indicator for remaining useful life prediction of bearings," Neurocomputing, vol. 240, pp. 98-109, 2017.

[7] C. Fangzhou, Q. Liyan, Q. Wei et al., "Enhanced particle filtering for bearing remaining useful life prediction of wind turbine drivetrain gearboxes," IEEE Transactions on Industrial Electronics, vol. 66, no. 6, p. 1, 2018.

[8] J. Wu, C. Zhang, Z. Chen, J. Wu, C. Zhang, and Z. Chen, “An online method for lithium-ion battery remaining useful life estimation using importance sampling and neural networks," Applied Energy, vol. 173, no. 1, pp. 134-140, 2016.

[9] P. Wang, Y. Tang, S. J. Bea, and Y. He, "Bayesian analysis of two-phase degradation data based on change-point wiener process," Reliability Engineering\& System Safety, vol. 170, pp. 244-256, 2018.

[10] D. Pan, J.-B. Liu, and J. Cao, "Remaining useful life estimation using an inverse gaussian degradation model," Neurocomputing, vol. 185, pp. 64-72, 2016.

[11] D. T. Liu, "Satellite lithium-on battery remaining cycle life prediction with novel indirect health indicator extraction," Energies, vol. 6, no. 8, pp. 3654-3668, 2013.

[12] J. X. Zhang, C. H. Hu, X. He et al., "Lifetime prognostics for deteriorating systems with time-varying random jumps," Reliability Engineering and System Safety, vol. 167, pp. 338350, 2017.

[13] L. Feng, H. Wang, and H. Zou, "A state-space-based prognostic model for hidden and age-dependent nonlinear diffusion degradation process," IEEE Transactions on Automation Science and Engineering, vol. 10, no. 4, pp. 1072-1086, 2013.

[14] Z. Si, Z. Xu, X. Ke, W. Wang, and Y. Sun, "Remaining useful life prediction for an adaptive skew-wiener process model," Mechanical Systems and Signal Processing, vol. 87, pp. 294306, 2017.

[15] H. Wang, "Lifetime prediction based on gamma processes from accelerated degradation data," Chinese Journal of Aeronautics, vol. 28, no. 1, pp. 172-179, 2015.

[16] L. A. Picón, L. C. M'endez-González, and M. I. Borbón, "Reliability estimation for products subjected to two-stage degradation tests based on a gamma convolution," Quality and Reliability Engineering International, vol. 32, no. 8, pp. 2901-2908, 2016.

[17] Z. Huang, Z. Xu, X. Ke et al., "Remaining useful life prediction for an adaptive skew-wiener process model," Mechanical Systems and Signal Processing, vol. 87, no. A, pp. 294-306, 2017.

[18] Z. X Zhang, "Degradation data analysis and remaining useful life estimation: a review on wiener-process-based methods," European Journal of Operational Research, vol. 271, no. 3, pp. 775-796, 2018.
[19] W. Peng, "Inverse Gaussian process models for degradation analysis: a bayesian perspective," Reliability Engineering \& System Safety, Reliability Engineering and System Safety, vol. 130, pp. 175-189, 2014.

[20] W. ZuoLi, Y.-J. Li, S.-P. Zhu, and H.-Z. Huang, "Bivariate analysis of incomplete degradation observations based on inverse gaussian processes and copulas," IEEE Transactions on Reliability, vol. 65, no. 2, pp. 624-639, 2016.

[21] Q. Zhai and Z. S. Ye, "RUL prediction of deteriorating products using an adaptive wiener process model," IEEE Transactions on Industrial Informatics, vol. 13, no. 6, pp. 2911-2921, 2017.

[22] N. Li, Y. Lei, L. Guo et al., "Remaining useful life prediction based on a general expression of stochastic process models," IEEE Transactions on Industrial Electronics, vol. 64, no. 7, pp. 5709-5718, 2017.

[23] H. Sun, J. Pan, J. Zhang, and D. Cao, "Non-linear wiener process-based cutting tool remaining useful life prediction considering measurement variability," The International Journal of Advanced Manufacturing Technology, vol. 107, no. 11-12, pp. 4493-4502, 2020.

[24] H. Liang, J. Q. Liu, Y. J. Gong et al., "Multi-phase residual life prediction of engines based on Wiener process," Journal of Beijing University of Aeronautics and Astronautics, vol. 44, no. 5, pp. 1081-1087, 2018, in Chinese.

[25] X. Wang, P. Jiang, B. Guo et al., "Real-time reliability evaluation for an individual product based on change-point gamma and wiener process," Quality and Reliability Engineering, vol. 30, no. 4, pp. 513-525, 2014.

[26] Z. Wang, Y. Wei, Q. Wu et al., "A two-stage degradation model considering the stage-varying of dispersity regulation," Quality \& Reliability Engineering International, vol. 35, no. 7, pp. 2115-2129, 2019.

[27] Z. X. Zhang, C. H. Hu, Y. B. Gao et al., "A residual useful life prediction approach for equipments with multi-state stochastic degradation," Journal of Systems Engineering, vol. 32, no. 1, pp. 1-7, 2017.

[28] W. A. Yan, B. Song, Z. Mao et al., "Empirical Bayesian estimation of wiener process with integrated degradation data and life data," in Proceedings of International Conference on Quality, pp. 183-188, IEEE, Chengdu, China, July 2013.

[29] Z. Huang, Z. Xu, W. Wang, and Y. Sun, "Remaining useful life prediction for a nonlinear heterogeneous wiener process model with an adaptive drift," IEEE Transactions on Reliability, vol. 64, no. 2, pp. 687-700, 2015.

[30] J. B. Ennis, F. W. Macdougall, R. A. Cooper et al., "Self-healing pulse capacitors for the national ignition facility (NIF)," in Proceedings of IEEE International Pulsed Power Conference, pp. 118-121, IEEE, Monterey, CA, USA, June 1999.

[31] B. H. Peng, J. L. Zhou, J. Feng et al., "Resudual lifetime prediction of metallized film pulse capacitors," Acta Electronica Sinica, vol. 39, no. 11, pp. 2674-2679, 2011, in Chinese.

[32] W. Wang, M. Carr, W. Xu, and K. Kobbacy, "A model for residual life prediction based on brownian motion with an adaptive drift," Microelectronics Reliability, vol. 51, no. 2, pp. 285-293, 2011. 